\title{
Instrumental performance and results from testing of the BLAST-TNG receiver, submillimeter optics, and MKID arrays
}

\author{
Nicholas Galitzki ${ }^{\mathrm{a}}$, Peter Ade ${ }^{\mathrm{b}}$, Francesco E. Angilè ${ }^{\mathrm{a}}$, Peter Ashtonc ${ }^{\mathrm{c}}$, Jason Austermann ${ }^{\mathrm{d}}$, \\ Tashalee Billings $^{\mathrm{a}}$, George Che ${ }^{\mathrm{e}}$, Hsiao-Mei Cho ${ }^{\mathrm{f}}$, Kristina Davis ${ }^{\mathrm{e}}$, Mark Devlin ${ }^{\mathrm{a}}$, Simon \\ Dicker $^{\mathrm{a}}$, Bradley J. Dober ${ }^{\mathrm{a}}$, Laura M. Fissel ${ }^{\mathrm{c}}$, Yasuo Fukui ${ }^{\mathrm{g}}$, Jiansong Gao ${ }^{\mathrm{d}}$, Samuel Gordon ${ }^{\mathrm{e}}$, \\ Christopher E. Groppi ${ }^{e}$, Seth Hillbrand ${ }^{a}$, Gene C. Hilton ${ }^{\mathrm{d}}$, Johannes Hubmayr ${ }^{\mathrm{d}}$, Kent D. \\ Irwin ${ }^{\mathrm{h}}$, Jeffrey Klein ${ }^{\mathrm{a}}$, Dale Li ${ }^{\mathrm{f}}$, Zhi-Yun Li ${ }^{\mathrm{i}}$, Nathan P. Lourie ${ }^{\mathrm{a}}$, Ian Lowe ${ }^{\mathrm{a}}$, Hamdi Mani ${ }^{\mathrm{e}}$, \\ Peter G. Martin ${ }^{\mathrm{j}}$, Philip Mauskopf ${ }^{\mathrm{e}}$, Christopher McKenney ${ }^{\mathrm{d}}$, Federico Nati ${ }^{\mathrm{a}}$, Giles Novak ${ }^{\mathrm{c}}$, \\ Enzo Pascale $^{\mathrm{b}}$, Giampaolo Pisano ${ }^{\mathrm{b}}$, Fabio P. Santos ${ }^{\mathrm{c}}$, Douglas Scott ${ }^{\mathrm{k}}$, Adrian Sinclair ${ }^{\mathrm{e}}$, Juan \\ D. Soler ${ }^{\mathrm{l}}$, Carole Tucker ${ }^{\mathrm{b}}$, Matthew Underhille, Michael Vissers ${ }^{\mathrm{d}}$, and Paul Williams ${ }^{\mathrm{c}}$ \\ ${ }^{a}$ University of Pennsylvania, 209 S 33rd St, Philadelphia, PA, USA \\ ${ }^{b}$ University of Cardiff, The Parade, Cardiff, United Kingdom \\ ${ }^{\mathrm{c}}$ Center for Interdisciplinary Exploration and Research in Astrophysics (CIERA) and \\ Department of Physics \& Astronomy, Northwestern University, 2145 Sheridan Road, Evanston, \\ IL 60208, USA \\ ${ }^{\mathrm{d}}$ National Institute of Standards and Technology, 325 Broadway, Boulder, CO, USA \\ eArizona State University, PO Box 871404, Tempe, AZ, USA \\ fSLAC National Accelerator Laboratory, 2575 Sand Hill Road, Menlo Park, CA, USA \\ 'Nagoya University, Furocho, Chikusa Ward, Nagoya, Aichi Prefecture, Japan \\ hStanford University, 382 Via Pueblo Mall, Stanford, CA, USA \\ ${ }^{\mathrm{i}}$ University of Virginia, 530 McCormick Road, Charlottesville, VA, USA \\ jUniversity of Toronto, 60 St. George Street, Toronto, ON, Canada \\ ${ }^{1}$ University of British Columbia, 6224 Agricultural Road, Vancouver, BC, Canada \\ ${ }^{k}$ Laboratoire AIM, Paris-Saclay, CEA/IRFU/SAp - CNRS - Université Paris Diderot, 91191, \\ Gif-sur-Yvette Cedex, France
}

\begin{abstract}
Polarized thermal emission from interstellar dust grains can be used to map magnetic fields in star forming molecular clouds and the diffuse interstellar medium (ISM). The Balloon-borne Large Aperture Submillimeter Telescope for Polarimetry (BLASTPol) flew from Antarctica in 2010 and 2012 and produced degree-scale polarization maps of several nearby molecular clouds with arcminute resolution. The success of BLASTPol has motivated a next-generation instrument, BLAST-TNG, which will use more than 3000 linear polarizationsensitive microwave kinetic inductance detectors (MKIDs) combined with a $2.5 \mathrm{~m}$ diameter carbon fiber primary mirror to make diffraction-limited observations at 250,350, and $500 \mu \mathrm{m}$. With 16 times the mapping speed of BLASTPol, sub-arcminute resolution, and a longer flight time, BLAST-TNG will be able to examine nearby molecular clouds and the diffuse galactic dust polarization spectrum in unprecedented detail. The $250 \mu \mathrm{m}$ detector array has been integrated into the new cryogenic receiver, and is undergoing testing to establish the optical and polarization characteristics of the instrument. BLAST-TNG will demonstrate the effectiveness of kilo-pixel MKID arrays for applications in submillimeter astronomy. BLAST-TNG is scheduled to fly from Antarctica in December 2017 for 28 days and will be the first balloon-borne telescope to offer a quarter of the flight for "shared risk" observing by the community.
\end{abstract}

E-mail: galitzki@sas.upenn.edu, Telephone: 12155737558 


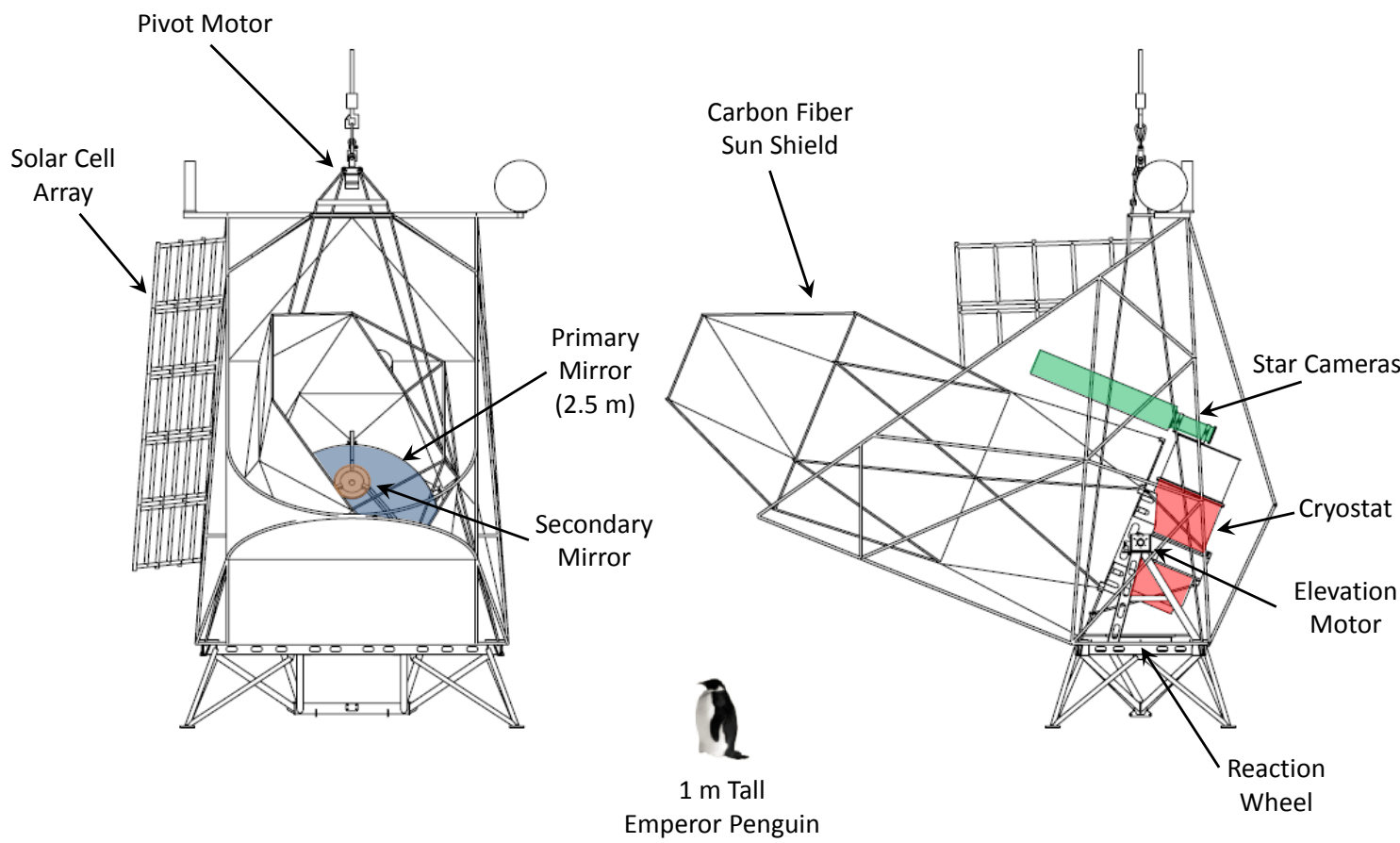

Figure 1. The front and side views of the BLAST-TNG telescope in its flight configuration. The cryostat, mirror optics bench, and star cameras are attached to an inner frame that moves in elevation. An extensive carbon fiber Sun shield also attaches to the inner frame to shield the optics at our closest pointing angle of $35^{\circ}$ to the sun.

Keywords: BLAST-TNG, Submillimeter, Polarimetry, MKIDs, Balloon-borne, Instrumentation, Star formation, Interstellar medium

\section{INTRODUCTION}

The Balloon-borne Large Aperture Submillimeter Telescope - The Next Generation (BLAST-TNG) ${ }^{1}$ consists of a $2.5 \mathrm{~m}$ Cassegrain telescope with three microwave kinetic inductance detector (MKID) arrays operating over $30 \%$ bandwidths centered on 250,350, and $500 \mu \mathrm{m}$, which have 1836, 950, 460 detectors with diffraction limited resolution of $25^{\prime \prime}, 35^{\prime \prime}$, and $50^{\prime \prime}$, respectively. It will be flown on a stratospheric balloon as part of NASA's long duration balloon program. An overview of the instrument design is shown in Figure 1.

The primary science goal of BLAST-TNG is to probe polarized thermal emission from dust in Galactic star forming regions in order to detect the local magnetic field orientation as projected on to the plane of the sky. The Antarctic flights in $2010^{2}$ and $2012^{3}$ of the previous instrument, BLASTPol, produced maps of magnetic field morphology in star forming regions with unprecedented levels of detail. ${ }^{4-10}$ The demonstrated scientific potential of submillimeter polarimetry observations prompted the design and construction of BLAST-TNG, which will have 16 times the BLASTPol mapping speed as well as better resolution.

The increased collecting area, sensitivity, and resolution will allow for the mapping of more clouds than the 2012 flight and enable observations of diffuse galactic dust emission. BLAST-TNG will allow us to investigate the role magnetic fields play in the formation and evolution of molecular clouds and their associated sub-structures including filaments, cores, and protostars as well as furthering our understanding of dust grain emission and alignment mechanisms. Additionally, BLAST-TNG provides a unique platform for observations which link the large-scale Planck $^{11}$ polarimetry maps to the small scale, but high resolution, polarimetry maps made by interferometers such as ALMA. ${ }^{12}$ 


\section{MAGNETIC FIELDS IN STAR-FORMING REGIONS}

In order to create a complete theory of star formation we must understand the processes that regulate the star formation rate in molecular clouds. Recent progress includes using observations of dust emission and extinction, which shows how core mass distribution correlates with observed stellar mass distribution. ${ }^{13}$ Herschel data has yielded many important results relevant to the star formation process such as observations that filamentary structures show a tendency for the densest filaments to be perpendicular to the local magnetic field, while faint, low density sub-filaments tend to be parallel to the local magnetic field. ${ }^{14}$ Additionally, Planck's all-sky maps of polarized dust emission have greatly increased our understanding of the role magnetic fields play in our galaxy. From these maps, strong evidence has been found that magnetic field orientation is correlated with molecular cloud structure and the degree of this alignment depends on the magnetic field strength and turbulent properties of the cloud being observed. ${ }^{15}$

However, there are many questions concerning the star-formation process and the evolution of cloud structure that remain to be addressed. ${ }^{16}$ Examples include whether the lifetimes of molecular clouds and their internal structures are equal ${ }^{17}$ to or larger ${ }^{18-20}$ than the turbulent crossing time. To have lifetimes longer than crossing times would require a supporting mechanism to counteract gravity. Magnetic fields could provide such support and numerical simulations have shown magnetic fields in clouds can drastically alter star formation efficiencies and the lifetimes of molecular clouds. ${ }^{21,22}$ However, knowledge of magnetic fields and their interaction with molecular cloud structure is still fairly limited. Zeeman splitting observations have produced measurements of the field strength along the line of sight, but are limited to bright regions, and optical extinction polarization observations have produced a small number of magnetic field pseudo-vectors, but only in areas of low extinction. ${ }^{23,24}$

The most promising method for detecting magnetic fields over large ranges of dust column density is with far-IR and submillimeter polarimetry. ${ }^{25-27}$ Spinning dust grains preferentially align with short axes parallel to the local magnetic field through the process of radiative alignment torques. ${ }^{28}$ The grains emit modified blackbody radiation that peaks in the far-IR/submillimeter and is polarized orthogonally to the local magnetic field. BLAST-TNG will have the unique capability to create degree-scale polarization maps of molecular clouds with sub-arcminute resolution with a mapping speed that will allow it to cover multiple targets during each flight. BLAST-TNG data will build on the results of BLASTPol, enabling additional and more detailed comparisons between polarization maps and numerical simulations. ${ }^{26}$

BLAST-TNG observations will target the following three key questions in star formation: i) Is core morphology and evolution determined by large-scale magnetic fields? ii) Does filamentary structure have a magnetic origin? iii) What is the field strength, and how does it vary from cloud to cloud? as further discussed in Ref. 29.

\section{INSTRUMENT}

BLAST-TNG will continue the legacy of BLASTPol with the construction of a new instrument that incorporates many successful elements from previous ballooning experiments. The optical layout of BLAST-TNG is shown in Figure 2 with the optical component parameters listed in Table 3. A $2.5 \mathrm{~m}$ diameter carbon fiber primary mirror* provides diffraction-limited observations. The telescope uses a Cassegrain configuration with the primary mirror illuminating a $56 \mathrm{~cm}$ diameter aluminum secondary mirror. The secondary mirror is actuated to allow for refocusing during the flight to adjust to the differential thermal contraction of the support structure. The beam is then re-imaged by the $4 \mathrm{~K}$ cold optics which use a modified Offner relay configuration. The light is split by two dichroic filters ${ }^{30}$ into the science bands at 250,350 , and $500 \mu \mathrm{m}$ to allow for simultaneous observations of the same patch of sky. The cold optics have been installed in the flight cryostat and will be characterized concurrently with the detector performance. The primary and secondary mirrors and supporting structure are on schedule for a July 2016 delivery.

Achieving a circular, 22' diameter, field of view (FOV) requires re-imaging optics significantly larger than those of BLASTPol. The size constraints necessitated the construction of a new cryostat which was also designed to have a longer hold time of $\sim 28$ days, versus the previous 13 day hold time of BLASTPol. The BLAST-TNG cryostat has a 250 liter liquid helium bath to provide cooling to $4 \mathrm{~K}$ and utilizes two vapor-cooled shields (VCS)

\footnotetext{
*Vanguard Space Technologies: 9431 Dowdy Drive, San Diego, CA 92126
} 


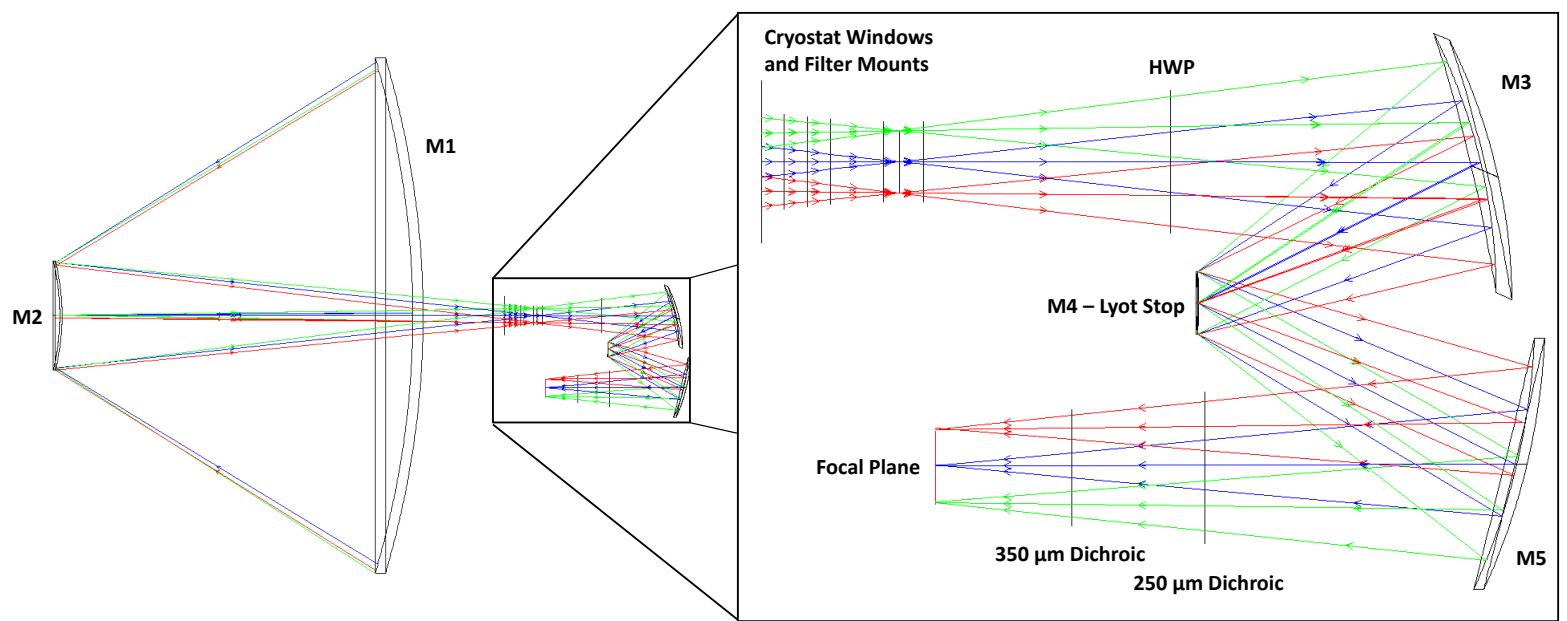

Figure 2. Side view of the optical configuration of BLAST-TNG with a detailed view of the $4 \mathrm{~K}$ cold optics. The telescope is an on axis Cassegrain that feeds into a modified Offner relay. M3, M4, and M5 are spherical mirrors with M4 acting as the Lyot stop for the telescope with a blackened hole that shadows the secondary mirror. Within the hole at the center of M4 is a calibrator lamp that provides an absolute calibration during flight operations to monitor responsivity drifts in the detectors. There are two dichroics that split the beam to the 250 and $350 \mu \mathrm{m}$ arrays. The $250 \mu \mathrm{m}$ dichroic is tilted at $22.5^{\circ}$ to the optical axis while the $350 \mu \mathrm{m}$ dichroic is tilted at $30^{\circ}$ to the optical axis. Only one of the three focal planes is shown. The half wave plate is inserted between the Cassegrain focus and M3.

Figure 3. Summary of BLAST-TNG Optics Characteristics

\begin{tabular}{lccccc}
\hline \hline Geometrical Charac. & M1 & M2 & M3 & M4 & M5 \\
\hline Nominal Shape & Paraboloid & Hyperboloid & Sphere & Sphere & Sphere \\
Conic Constant & -1.0 & -2.38 & 0.000 & 0.000 & 0.000 \\
Radius of Curvature & $4.132 \mathrm{~m}$ & $1.210 \mathrm{~m}$ & $655.6 \mathrm{~mm}$ & $376.5 \mathrm{~mm}$ & $749.4 \mathrm{~mm}$ \\
Aperture & $\varnothing 2.5 \mathrm{~m}$ & $\varnothing 0.573 \mathrm{~m}$ & $\varnothing 28 \mathrm{~cm}$ & $\varnothing 7 \mathrm{~cm}$ & $\varnothing 28 \mathrm{~cm}$ \\
\hline
\end{tabular}

to provide additional thermal isolation. The arrays are kept at $270 \mathrm{mK}$ by a closed-cycle ${ }^{3} \mathrm{He}$ refrigerator with a $1 \mathrm{~K}$ intercept stage provided by an open cycle ${ }^{4} \mathrm{He}$ pumped pot that is fed off the main Helium tank and vents to atmosphere during flight. The detector arrays and cold optics are contained within an Amuneal ${ }^{\dagger}$ enclosure which provides shielding from ambient magnetic fields. The light is coupled to the detectors with an aluminum feedhorn block with a three step Potter ${ }^{31}$ style profile to provide a more symmetric beam in both polarization directions than previously achieved with conical feedhorns. ${ }^{32}$ Each feedhorn pixel contains two orthogonally oriented MKIDs (See Fig. 4). BLAST-TNG will serve as a pathfinder instrument for MKIDs, ${ }^{33}$ which have never been flown before. The development of MKID arrays for astronomy is an extremely active area of detector research, and flight testing them will be a significant milestone.

Additional details of the instrument design including the electronics, pointing system, control system, and detector readout can be found in Ref. 1.

\footnotetext{
${ }^{\dagger}$ Amuneal Manufacturing Corp., 4737 Darrah Street, Philadelphia, PA 19124
} 


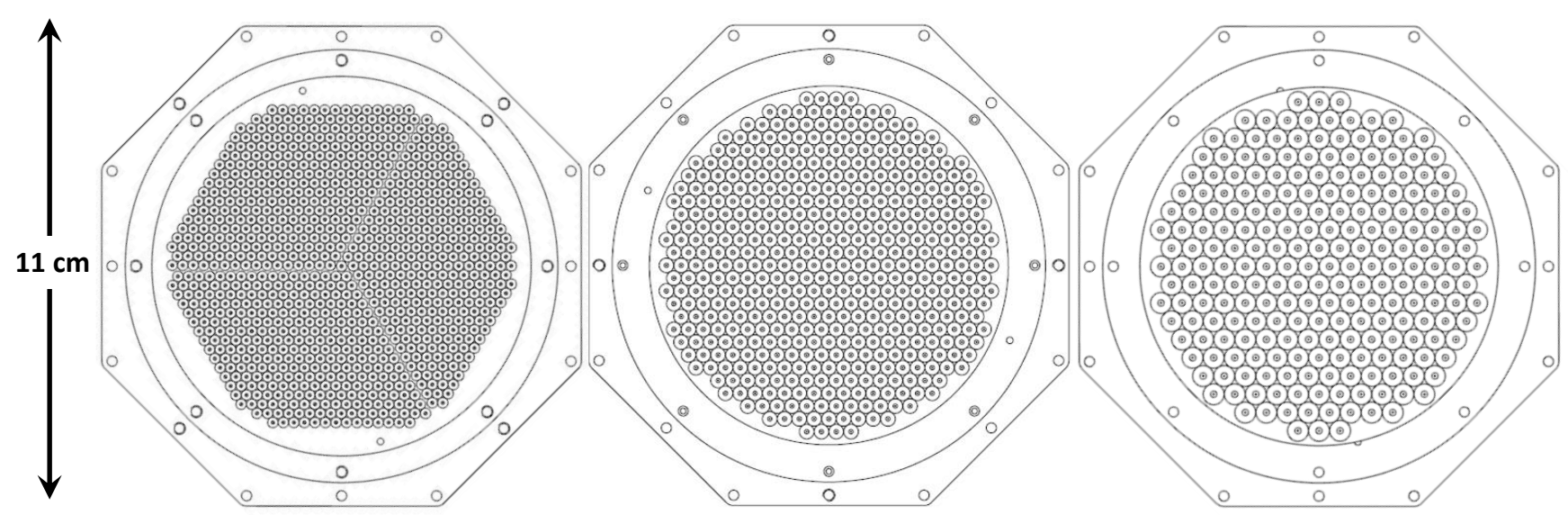

Figure 4. A top image of the feedhorn block designs corresponding to the $250,350,500 \mu \mathrm{m}$ arrays (from left to right) with 759, 475, 230 pixels, respectively. Each pixel contains two orthogonally oriented MKIDs.

\subsection{Cryogenic Performance}

The BLAST-TNG cryostat ${ }^{\ddagger}$ was delivered to the University of Pennsylvania (UPenn) in February 2015. Over the following year the thermometry, refrigerator systems, re-imaging optics, and the $250 \mu \mathrm{m}$ detector array were integrated and tested in increments to allow us to characterize the performance of the cryogenic system and debug systems as they were added. The cross section of the cryostat can be seen in Figure 5. A thermal model was developed to describe the expected steady-state thermal load on each temperature stage. The thermal model took into account radiative loading as well as conduction through the G10 mechanical supports, cabling, motor axles, the helium fill tube, and the $1 \mathrm{~K}$ refrigerator exhaust tube (See Table 6).

During testing, the VCSs were observed to reach a steady temperature of $\sim 65 \mathrm{~K}$ and $\sim 165 \mathrm{~K}$. The steady state load on the $4 \mathrm{~K}$ cold plate was measured to be $\sim 340 \mathrm{~mW}$, as determined by the measured helium boil-off rate of the cryostat. The loading corresponds to a 22.5 day hold time for the 250 liter tank with an approximate boil-off rate of 11 liters of liquid helium per day. The observed loading is approximately $40 \%$ larger than the predicted loading of $240 \mathrm{~mW}$ from the thermal model used in the design of the cryostat. We believe our excess loading is due in part to un-modeled light leaks in the MLI blanket around fixtures and feedthroughs, as suggested by SPIDER ${ }^{34}$ but also due to unexpected complexities in the thermal behavior of G10 material, which provides the mechanical support in the BLAST-TNG cryostat.

By adjusting the conductive model of the G10 we can account for most of the additional loading observed. This observation has prompted us to replace the G10 sections between the $165 \mathrm{~K}$ and $65 \mathrm{~K}$ stages and between the $65 \mathrm{~K}$ and $4 \mathrm{~K}$ stages with thinner walled material. The change will decrease the effective loading on the $4 \mathrm{~K}$ stage to bring the performance in line with our target 28 day hold time. It should also be noted from Ref. 34 that the thermal loading at balloon flight altitudes is observed to be less due to the cooler temperature of the cryogenic vessel and the reduced optical loading through the window which can increase the hold times of cryostats during flight.

As a baseline for comparing cryostat performance a figure of merit was developed in Ref. 35 that divides the radiative loading, $H$, by the cryogen depletion rate, $R$. For the SPIDER cryostat along with many satellite cryostats, $H / R \approx 60 \mathrm{~W}$ Days/L whereas the BLAST-TNG cryostat ranks slightly higher with $H / R \approx$ $230 \mathrm{~W}$ Days/L using the $250 \mathrm{~L}$ cryogenic volume, a hold time of 22.5 days, and the approximately $6 \mathrm{~m}^{2}$ surface area of the $4 \mathrm{~K}$ shield.

\subsection{Detector Performance}

The $250 \mu \mathrm{m}$ MKID detectors have been shown to be photon noise limited in a 7 pixel test array that was feedhorn coupled to a variable blackbody source. Additional details of the tests and results can be found in

\footnotetext{
${ }^{\ddagger}$ Precision Cryogenic Systems Inc., 7804 Rockville Rd., Indianapolis, IN 46214
} 


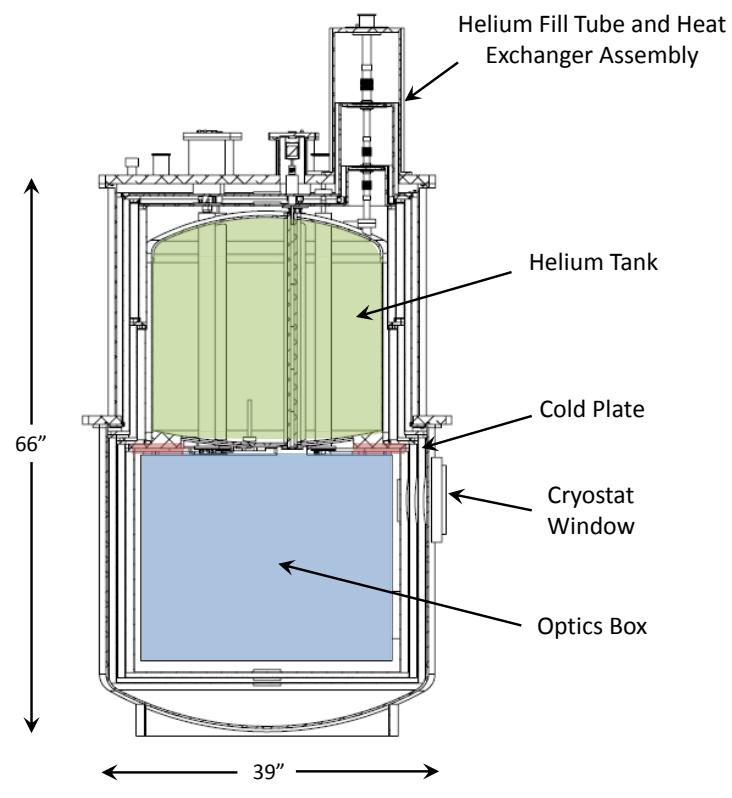

Figure 5. Cross section view of the BLAST-TNG cryostat. The liquid helium cryostat includes two helium vapor heat exchangers that cool two shields to provide thermal isolation of the cold optics. The inner vapor cooled shield is kept at $\sim 65 \mathrm{~K}$ and the outer one at $\sim 165 \mathrm{~K}$. The cryostat has a predicted hold time of 28 days.

Figure 6. List of the percentages of primary contributions to the thermal load at each of the main stages

\begin{tabular}{lccccc}
\hline \hline & Radiative & Conductive & Plumbing & Cables & Misc. \\
\hline VCS2 Stage (165 K) & $77 \%$ & $16 \%$ & $6 \%$ & $1 \%$ & $<1 \%$ \\
VCS1 Stage (65 K) & $22 \%$ & $50 \%$ & $22 \%$ & $6 \%$ & $<1 \%$ \\
LHe Stage (4 K) & $24 \%$ & $50 \%$ & $11 \%$ & $<1 \%$ & $14 \%$ \\
& & & & & \\
\hline
\end{tabular}

Ref. 36. The noise equivalent power (NEP) of the detectors was fit using a three component noise model that included recombination noise and the photon noise on top of a flat background noise level. The fit determined that the detectors were limited by photon noise in the range $1 \mathrm{pW}$ to $20 \mathrm{pW}$, which comfortably encompasses the expected BLAST-TNG flight loading on the detectors of $\sim 5$ to $\sim 14 \mathrm{pW}$. The fit to the noise model also produces an estimate of the optical coupling efficiency which was determined to be $\sim 70 \%$.

The $250 \mu \mathrm{m}(1.2 \mathrm{THz})$ array is designed to have a bandpass from 325 to $175 \mu \mathrm{m}(1.0$ to $1.4 \mathrm{THz})$ which is defined on the long wavelength end by the feedhorn profile and on the short wavelength end, by a low pass filter mounted in front of the feedhorn block. The bandpass was tested using a hot $1050^{\circ} \mathrm{C}$ thermal source coupled to a Fourier Transform Spectrometer (FTS) that filled the feedhorn beam. The tests determined that the edges of the bandpass for both polarization directions in a single pixel to be in agreement with the design of the filters and feedhorns. ${ }^{37}$

The MKID's polarization performance has also been tested and is described in detail in Ref. 37. The tests were performed with a source chopped between $1050^{\circ} \mathrm{C}$ and $20^{\circ} \mathrm{C}$ with a wire grid polarizer mounted between the source and the detectors. The polarizing grid is then rotated to produce a sinusoidal response at the detectors 

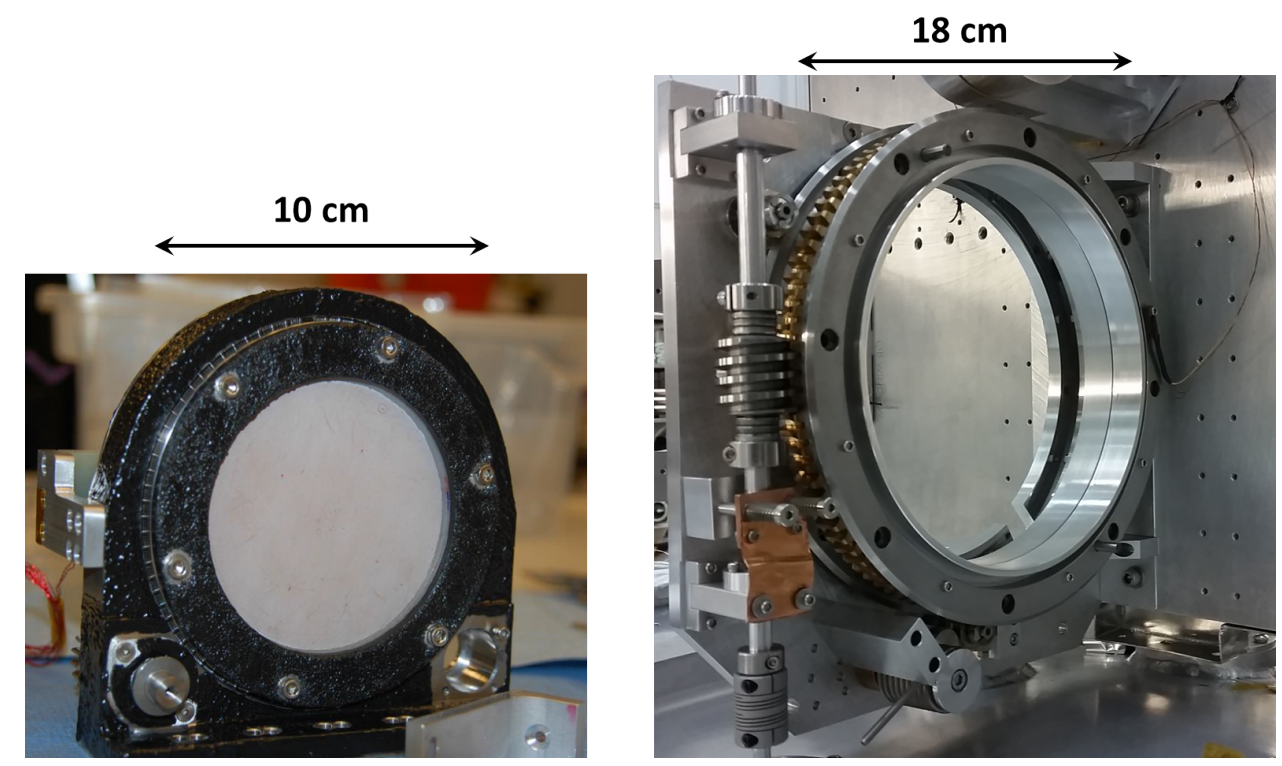

Figure 7. Left: An image of the half wave plate and rotator mechanism that was used in BLASTPol. ${ }^{41}$ Right: An image of the new HWP rotator mechanism with a larger clear aperture of $\sim 18 \mathrm{~cm}$. The rotator is bolted to the optics bench which is in turn mounted on the cold plate of the helium tank. Rotation of the HWP is driven by a worm gear that is coupled to a motor mounted to the $300 \mathrm{~K}$ lid of the cryostat with a G10 shaft to provide thermal isolation.

with orthogonal detectors 90 degrees out of phase. Fits to these measurements determined the cross-polar signal for both detector directions in a pixel to be $<3 \%$, demonstrating the effectiveness of feedhorn coupled MKIDs for submillimeter polarimetry measurements.

Additional details of the MKID design and performance can be found in Ref. 38.

\subsection{Readout Electronics}

The BLAST-TNG readout is a highly multiplexed 1024 channel digital spectrometer which is the first of its kind to have been developed for the second generation Reconfigurable Open Architecture Computing Hardware (ROACH-2). ${ }^{39}$ The ROACH-2 architecture is based on a field programmable gate array (FPGA), and is a product of the Collaboration for Astronomy Signal Processing and Electronics Research (CASPER). The ROACH is used in conjunction with a DAC/ADC board that was designed for ARCONS. ${ }^{40}$ Building on the legacy of previous KID readouts created for the ROACH platform, the BLAST-TNG firmware performs coarse and fine channelization of $512 \mathrm{MHz}$ of RF bandwidth. The resulting channels are $\sim 100 \mathrm{kHz}$ wide and can be read out at rates of $200 \mathrm{~Hz}$ to $500 \mathrm{kHz}$. BLAST-TNG uses five ROACH-2 boards, three for the $250 \mu \mathrm{m}$ array and one each for the 350 and $500 \mu \mathrm{m}$ arrays, along with a suite of IF components and single board computers. The readout hardware is housed in the 'ROACH-2 Motel', a custom enclosure designed for BLAST-TNG. Control software is written in Python, and will be ported to $\mathrm{C}$ for integration into the flight software. Details of the systems overall performance are forthcoming.

\subsection{Polarimetry}

The primary scanning strategy during flight will be a slow raster scan, which works well for extended sources. A typical BLAST-TNG raster will scan across targets in azimuth at a speed of approximately $0.5^{\circ} \mathrm{s}^{-1}$ with an elevation scan speed calculated to change the elevation by $1 / 3$ the array FOV in one crossing of the target in azimuth. A Half Wave Plate (HWP) is used to modulate the polarization signal, so that each pixel samples $I$, $Q$, and $U$ multiple times during the course of a scan which allows us to control for polarization systematics. The HWP is stepped between four set angles $\left(0^{\circ}, 22.5^{\circ}, 45^{\circ}\right.$, and $\left.67.5^{\circ}\right)$ after each completed scan of a source in elevation. The HWP used in BLASTPol ${ }^{41}$ had a $10 \mathrm{~cm}$ diameter aperture and was made from five layers of $500 \mu \mathrm{m}$ thick sapphire with an anti-reflective coating. However, the technology did not scale easily to larger apertures 
necessitating a new approach. The HWP developed for BLAST-TNG uses metal mesh filter technology ${ }^{30}$ to make a large diameter, $\sim 18 \mathrm{~cm}$, HWP (See Fig. 7). These types of HWP have demonstrated broad-band transmission at $\mathrm{THz}$ frequencies. ${ }^{42,43}$

The detector rows are oriented parallel to the nominal scan direction with the MKID orientation rotated by 45 degrees in adjacent pixels along the scan direction. This alignment allows for sampling of both $Q$ and $U$ Stokes parameter on a timescale that is shorter than the array's common-mode $1 / f$ noise, creating a redundant polarization modulation feature. The sampling timescale of the Stokes parameter is $\sim 0.025 \mathrm{~s}$, which is determined by the detector separation, $\sim 45^{\prime \prime}$ at $250 \mu \mathrm{m}$, and typical scan speed, $\sim 0.5^{\circ} \mathrm{s}^{-1}$.

\section{CONCLUSION}

BLASTPol successfully demonstrated the viability of balloon-borne telescopes to explore the polarization of the submillimeter sky and has led directly to the development of BLAST-TNG. Progress in the construction of the new instrument is on schedule and is proceeding rapidly. The cryogenics system has been thoroughly tested and is in a near flight ready status. Additionally, the detector technology is well understood and has undergone extensive testing at NIST prior to delivery to UPenn. The $250 \mu \mathrm{m}$ array has been installed in the cryostat and has been successfully incorporated into the readout architecture with observed optical sensitivity during cryogenic operations. We expect to demonstrate the detector's target sensitivity in the flight cryostat during the summer of 2016. The 350 and $500 \mu \mathrm{m}$ arrays and feedhorn blocks are being fabricated for testing and installation by fall 2016. The new BLAST-TNG instrument will give us magnetic field morphology maps at unprecedented resolution that span entire molecular cloud structures, allowing us to link the full-sky polarimetry maps of Planck with the high-resolution, small-area polarimetry maps, of telescopes such as ALMA. This will provide strong constraints on models of magnetic fields and turbulent interaction within magnetic fields as well as increasing our understanding of dust grain models.

\section{ACKNOWLEDGMENTS}

BLAST is funded by NASA through grant number NNX13AE50G S08. Detector development is supported in part by NASA through NNH13ZDA001N-APRA. Brad Dober was funded by a NASA Earth and Space Science Fellowship NNX12AL58H S02. The BLAST-TNG collaboration would like to acknowledge the Xilinx University Program for their generous donation of five Virtex-6 FPGAs for use in our ROACH-2 readout electronics. Peter Ashton was supported through Reach for the Stars, a GK-12 program supported by the National Science Foundation under grant DGE-0948017. We would also like to thank the Columbia Scientic Balloon Facility (CSBF) staff for their continued outstanding work.

\section{REFERENCES}

[1] Galitzki, N., Ade, P. A. R., Angilè, F. E., Ashton, P., Beall, J. A., Becker, D., Bradford, K. J., Che, G., Cho, H.-M., Devlin, M. J., Dober, B. J., Fissel, L. M., Fukui, Y., Gao, J., Groppi, C. E., Hillbrand, S., Hilton, G. C., Hubmayr, J., Irwin, K. D., Klein, J., van Lanen, J., Li, D., Li, Z.-Y., Lourie, N. P., Mani, H., Martin, P. G., Mauskopf, P., Nakamura, F., Novak, G., Pappas, D. P., Pascale, E., Pisano, G., Santos, F. P., Savini, G., Scott, D., Stanchfield, S., Tucker, C., Ullom, J. N., Underhill, M., Vissers, M. R., and WardThompson, D., "The Next Generation BLAST Experiment," Journal of Astronomical Instrumentation 3, 1440001 (2014).

[2] Pascale, E., Ade, P. A. R., Angilè, F. E., Benton, S. J., Chapin, E. L., Devlin, M. J., Fissel, L. M., Gandilo, N. N., Gundersen, J. O., Hargrave, P. C., Hughes, D. H., Klein, J., Korotkov, A. L., Marsden, G., Matthews, T. G., Moncelsi, L., Mroczkowski, T. K., Netterfield, C. B., Novak, G., Olmi, L., Savini, G., Scott, D., Shariff, J. A., Soler, J. D., Thomas, N. E., Truch, M. D. P., Tucker, C. E., Tucker, G. S., Ward-Thompson, D., and Wiebe, D. V., "The balloon-borne large-aperture submillimeter telescope for polarimetry - BLASTPol: performance and results from the 2010 Antarctic flight," in [Society of PhotoOptical Instrumentation Engineers (SPIE) Conference Series], Proceedings of SPIE (2012). 
[3] Galitzki, N., Ade, P. A. R., Angilè, F. E., Benton, S. J., Devlin, M. J., Dober, B., Fissel, L. M., Fukui, Y., Gandilo, N. N., Klein, J., Korotkov, A. L., Matthews, T. G., Moncelsi, L., Netterfield, C. B., Novak, G., Nutter, D., Pascale, E., Poidevin, F., Savini, G., Scott, D., Shariff, J. A., Soler, J. D., Tucker, C. E., Tucker, G. S., and Ward-Thompson, D., "The Balloon-borne Large Aperture Submillimeter Telescope for Polarimetry-BLASTPol: Performance and results from the 2012 Antarctic flight," in [Ground-based and Airborne Telescopes V], Presented at the Society of Photo-Optical Instrumentation Engineers (SPIE) Conference $\mathbf{9 1 4 5}$ (June 2014).

[4] Matthews, T. G., Ade, P. A. R., Angilè, F. E., Benton, S. J., Chapin, E. L., Chapman, N. L., Devlin, M. J., Fissel, L. M., Fukui, Y., Gandilo, N. N., Gunderson, J. O., Hargrave, P. C.and Klein, J., Korotkov, A. L., Moncelsi, L., K., M. T., Netterfield, C. B., Novak, G., Nutter, D. Olmi, L., Pascale, E., Poidevin, F., Savini, G., Scott, D., Shariff, J. A., Soler, J. D., Tachihara, K., Thomas, N. E., Truch, M. D. P., Tucker, C. E., Tucker, G. S., and Ward-Thompson, D., "Lupus I Observations from the 2010 Flight of the Balloon-borne Large Aperture Submillimeter Telescope for Polarimetry," ApJ 784, 822-833 (Mar. 2014).

[5] Gandilo, N. N., Ade, P. A. R., Angilè, F. E., Ashton, P., Benton, S. J., Devlin, M. J., Dober, B., Fissel, L. M., Fukui, Y., Galitzki, N., Klein, J., Korotkov, A. L., Li, Z.-Y., Martin, P. G., Matthews, T. G., Moncelsi, L., Nakamura, F., Netterfield, C. B., Novak, G., Pascale, E., Poidevin, F., Santos, F. P., Savini, G., Scott, D., Shariff, J. A., Diego Soler, J., Thomas, N. E., Tucker, C. E., Tucker, G. S., and Ward-Thompson, D., "Submillimeter Polarization Spectrum in the Vela C Molecular Cloud," ArXiv e-prints (Dec. 2015).

[6] Fissel, L. M., Ade, P. A. R., Angilè, F. E., Ashton, P., Benton, S., Devlin, M. J., Dober, B., Fukui, Y., Galitzki, N., Gandilo, N. N., Klein, J. R., Li, Z.-Y., Korotkov, A. L., Martin, P. G., Matthews, T. G., Moncelsi, L., Nakamura, F., Barth Netterfield, C., Novak, G., Pascale, E., Poidevin, F., Santos, F. P., Savini, G., Scott, D., Shariff, J. A., Soler, J. D., Thomas, N. E., Tucker, C. E., Tucker, G. S., and Ward-Thompson, D., "Balloon-Borne Submillimeter Polarimetry of the Vela C Molecular Cloud: Systematic Dependence of Polarization Fraction on Column Density and Local Polarization-Angle Dispersion," ArXiv e-prints (Sept. 2015).

[7] Santos, F. P., Ade, P. A. R., Angile, F. E., Ashton, P., Benton, S. J., Devlin, M. J., Dober, B., Fissel, L. M., Fukui, Y., Galitzki, N., Gandilo, N. N., Klein, J., Korotkov, A. L., Li, Z.-Y., Martin, P. G., Matthews, T. G., Moncelsi, L., Nakamura, F., Netterfield, C. B., Novak, G., Pascale, E., Poidevin, F., Savini, G., Scott, D., Shariff, J. A., Diego Soler, J., Thomas, N. E., Tucker, C. E., Tucker, G. S., and Ward-Thompson, D., "Comparing submillimeter polarized emission with near-infrared polarization of background stars for the Vela C molecular cloud," ArXiv e-prints (May 2016).

[8] Soler, J. D., Ade, P. A. R., Angile, F. E., Ashton, P., Benton, S. J., Devlin, M. J., Dober, B., Fissel, L. M., Fukui, Y., Galitzki, N., Gandilo, N. N., Klein, J., Korotkov, A. L., Li, Z.-Y., Martin, P. G., Matthews, T. G., Moncelsi, L., Nakamura, F., Netterfield, C. B., Novak, G., Pascale, E., Poidevin, F., Santos, F. P., Savini, G., Scott, D., Shariff, J. A., Thomas, N. E., Tucker, C. E., Tucker, G. S., and Ward-Thompson, D. To be submitted to ApJ (In preparation).

[9] Galitzki, N., Ade, P. A. R., Angile, F. E., Ashton, P., Benton, S. J., Devlin, M. J., Dober, B., Fissel, L. M., Fukui, Y., Gandilo, N. N., Klein, J., Korotkov, A. L., Li, Z.-Y., Martin, P. G., Matthews, T. G., Moncelsi, L., Nakamura, F., Netterfield, C. B., Novak, G., Pascale, E., Poidevin, F., Santos, F. P., Savini, G., Scott, D., Shariff, J. A., Soler, J. D., Thomas, N. E., Tucker, C. E., Tucker, G. S., and Ward-Thompson, D. To be submitted to ApJ (In preparation).

[10] Shariff, J., Ade, P. A. R., Angile, F. E., Ashton, P., Benton, S. J., Devlin, M. J., Dober, B., Fissel, L. M., Fukui, Y., Galitzki, N., Gandilo, N. N., Klein, J., Korotkov, A. L., Li, Z.-Y., Martin, P. G., Matthews, T. G., Moncelsi, L., Nakamura, F., Netterfield, C. B., Novak, G., Pascale, E., Poidevin, F., Santos, F. P., Savini, G., Scott, D., Shariff, J. A., Soler, J. D. Thomas, N. E., Tucker, C. E., Tucker, G. S., and Ward-Thompson, D. To be submitted to ApJ (In preparation).

[11] Planck Collaboration, Adam, R., Ade, P. A. R., Aghanim, N., Akrami, Y., Alves, M. I. R., Arnaud, M., Arroja, F., Aumont, J., Baccigalupi, C., and et al., "Planck 2015 results. I. Overview of products and scientific results," ArXiv e-prints (Feb. 2015).

[12] Cortes, P. C., Girart, J. M., Hull, C., Sridharan, T. K., Louvet, F., Plambeck, R., Li, Z.-Y., Crutcher, R. M., and Lai, S.-P., "Interferometric mapping of magnetic fields: The ALMA view of the massive star forming clump W43-MM1," ArXiv e-prints (May 2016). 
[13] Nutter, D. and Ward-Thompson, D., "A SCUBA survey of Orion - the low-mass end of the core mass function," MNRAS 374, 1413-1420 (Feb. 2007).

[14] Palmeirim, P., André, P., Kirk, J., Ward-Thompson, D., Arzoumanian, D., Könyves, V., Didelon, P., Schneider, N., Benedettini, M., Bontemps, S., Di Francesco, J., Elia, D., Griffin, M., Hennemann, M., Hill, T., Martin, P. G., Men'shchikov, A., Molinari, S., Motte, F., Nguyen Luong, Q., Nutter, D., Peretto, N., Pezzuto, S., Roy, A., Rygl, K. L. J., Spinoglio, L., and White, G. L., "Herschel view of the Taurus B211/3 filament and striations: evidence of filamentary growth?," A\&A 550, A38 (Feb. 2013).

[15] Planck Collaboration, Ade, P. A. R., Aghanim, N., Alina, D., Alves, M. I. R., Armitage-Caplan, C., Arnaud, M., Arzoumanian, D., Ashdown, M., Atrio-Barandela, F., and et al., "Planck intermediate results. XXXV. Probing the role of the magnetic field in the formation of structure in molecular clouds," A\&A 586, A138 (Feb. 2016).

[16] McKee, C. F. and Ostriker, E. C., "Theory of Star Formation," ARA\&A 45, 565-687 (Sept. 2007).

[17] Vázquez-Semadeni, E., Ryu, D., Passot, T., González, R. F., and Gazol, A., "Molecular Cloud Evolution. I. Molecular Cloud and Thin Cold Neutral Medium Sheet Formation," ApJ 643, 245-259 (May 2006).

[18] Netterfield, C. B., Ade, P. A. R., Bock, J. J., Chapin, E. L., Devlin, M. J., Griffin, M., Gundersen, J. O., Halpern, M., Hargrave, P. C., Hughes, D. H., Klein, J., Marsden, G., Martin, P. G., Mauskopf, P., Olmi, L., Pascale, E., Patanchon, G., Rex, M., Roy, A., Scott, D., Semisch, C., Thomas, N., Truch, M. D. P., Tucker, C., Tucker, G. S., Viero, M. P., and Wiebe, D. V., "BLAST: The Mass Function, Lifetimes, and Properties of Intermediate Mass Cores from a $50 \mathrm{deg}^{2}$ Submillimeter Galactic Survey in Vela $\ell \approx 265 \mathrm{deg}$ )," ApJ 707, 1824-1835 (Dec. 2009).

[19] Blitz, L., Fukui, Y., Kawamura, A., Leroy, A., Mizuno, N., and Rosolowsky, E., "Giant Molecular Clouds in Local Group Galaxies," Protostars and Planets V 5, 81-96 (2007).

[20] Goldsmith, P. F., Heyer, M., Narayanan, G., Snell, R., Li, D., and Brunt, C., "Large-Scale Structure of the Molecular Gas in Taurus Revealed by High Linear Dynamic Range Spectral Line Mapping," ApJ 680, 428-445 (June 2008).

[21] Li, Z.-Y., Wang, P., Abel, T., and Nakamura, F., "Lowering the Characteristic Mass of Cluster Stars by Magnetic Fields and Outflow Feedback," ApJ 720, L26-L30 (Sept. 2010).

[22] Hennebelle, P., Commerçon, B., Joos, M., Klessen, R. S., Krumholz, M., Tan, J. C., and Teyssier, R., "Collapse, outflows and fragmentation of massive, turbulent and magnetized prestellar barotropic cores," A\&A 528, A72 (Apr. 2011).

[23] Crutcher, S. H. and Osei, A. J., "Spatial Beam Propagation with a Perturbation in a Nonlinear Medium with Power Law," in [American Institute of Physics Conference Series], Oluseyi, H. M., ed., American Institute of Physics Conference Series 1280, 130-141 (Oct. 2010).

[24] Falgarone, E., Troland, T. H., Crutcher, R. M., and Paubert, G., "CN Zeeman measurements in star formation regions," A\&A 487, 247-252 (Aug. 2008).

[25] Hildebrand, R. H., Davidson, J. A., Dotson, J. L., Dowell, C. D., Novak, G., and Vaillancourt, J. E., "A Primer on Far-Infrared Polarimetry," PASP 112, 1215-1235 (Sept. 2000).

[26] Ward-Thompson, D., Kirk, J. M., Crutcher, R. M., Greaves, J. S., Holland, W. S., and André, P., "First Observations of the Magnetic Field Geometry in Prestellar Cores," ApJ 537, L135-L138 (July 2000).

[27] Ward-Thompson, D., Sen, A. K., Kirk, J. M., and Nutter, D., "Optical and submillimetre observations of Bok globules - tracing the magnetic field from low to high density," MNRAS 398, 394-400 (Sept. 2009).

[28] Andersson, B.-G., Lazarian, A., and Vaillancourt, J. E., "Interstellar Dust Grain Alignment," ARA\&A 53, 501-539 (Aug. 2015).

[29] Fissel, L. M., Ade, P. A. R., Angilè, F. E., Benton, S. J., Chapin, E. L., Devlin, M. J., Gandilo, N. N., Gundersen, J. O., Hargrave, P. C., Hughes, D. H., Klein, J., Korotkov, A. L., Marsden, G., Matthews, T. G., Moncelsi, L., Mroczkowski, T. K., Netterfield, C. B., Novak, G., Olmi, L., Pascale, E., Savini, G., Scott, D., Shariff, J. A., Soler, J. D., Thomas, N. E., Truch, M. D. P., Tucker, C. E., Tucker, G. S., Ward-Thompson, D., and Wiebe, D. V., "The balloon-borne large-aperture submillimeter telescope for polarimetry: BLASTPol," in [Society of Photo-Optical Instrumentation Engineers (SPIE) Conference Series], Proceedings of SPIE 7741 (July 2010). 
[30] Ade, P. A. R., Pisano, G., Tucker, C., and Weaver, S., "A review of metal mesh filters," in [Society of PhotoOptical Instrumentation Engineers (SPIE) Conference Series], Proceedings of SPIE 6275 (July 2006).

[31] Potter, P. D., "A New Horn Antenna with Suppressed Sidelobes and Equal Beamwidths," Microwave Journal , 71-78 (June 1963).

[32] Rownd, B., Bock, J. J., Chattopadhyay, G., Glenn, J., and Griffin, M. J., "Design and performance of feedhorn-coupled bolometer arrays for SPIRE," in [Society of Photo-Optical Instrumentation Engineers (SPIE) Conference Series], Proceedings of SPIE 4855, 510-519 (Feb. 2003).

[33] Day, P. K., LeDuc, H. G., Mazin, B. A., Vayonakis, A., and Zmuidzinas, J., "A broadband superconducting detector suitable for use in large arrays," Nature 425, 817-821 (Oct. 2003).

[34] Gudmundsson, J. E., Ade, P. A. R., Amiri, M., Benton, S. J., Bock, J. J., Bond, J. R., Bryan, S. A., Chiang, H. C., Contaldi, C. R., Crill, B. P., Dore, O., Filippini, J. P., Fraisse, A. A., Gambrel, A., Gandilo, N. N., Hasselfield, M., Halpern, M., Hilton, G., Holmes, W., Hristov, V. V., Irwin, K. D., Jones, W. C., Kermish, Z., MacTavish, C. J., Mason, P. V., Megerian, K., Moncelsi, L., Montroy, T. E., Morford, T. A., Nagy, J. M., Netterfield, C. B., Rahlin, A. S., Reintsema, C. D., Ruhl, J. E., Runyan, M. C., Shariff, J. A., Soler, J. D., Trangsrud, A., Tucker, C., Tucker, R. S., Turner, A. D., Wiebe, D. V., and Young, E., "The thermal design, characterization, and performance of the SPIDER long-duration balloon cryostat," Cryogenics $\mathbf{7 2}$, 65-76 (Dec. 2015).

[35] Holmes, W., Cho, H., Hahn, I., Larson, M., Schweickart, R., and Volz, S., "Performance comparisons of space borne cryostats," Cryogenics 41, 865-870 (Nov. 2001).

[36] Hubmayr, J., Beall, J., Becker, D., Cho, H.-M., Devlin, M., Dober, B., Groppi, C., Hilton, G. C., Irwin, K. D., Li, D., Mauskopf, P., Pappas, D. P., Van Lanen, J., Vissers, M. R., Wang, Y., Wei, L. F., and Gao, J., "Photon-noise limited sensitivity in titanium nitride kinetic inductance detectors," Applied Physics Letters 106, 073505 (Feb. 2015).

[37] Dober, B., Austermann, J. A., Beall, J. A., Becker, D., Che, G., Cho, H. M., Devlin, M., Duff, S. M., Galitzki, N., Gao, J., Groppi, C., Hilton, G. C., Hubmayr, J., Irwin, K. D., McKenney, C. M., Li, D., Lourie, N., Mauskopf, P., Vissers, M. R., and Wang, Y., "Optical Demonstration of THz, Dual-Polarization Sensitive Microwave Kinetic Inductance Detectors," Journal of Low Temperature Physics (Dec. 2015).

[38] McKenney, C. M., Ade, P., Angilè, F. E., Ashton, P. A., Austermann, J. A., Beall, J. A., Becker, D., Che, G., Cho, H. M., Devlin, M., Dober, B., Duff, S. M., Galitzki, N., Gao, J., Groppi, C., Hilton, G. C., Hubmayr, J., Irwin, K. D., McKenney, C. M., Li, D., Lourie, N., Mauskopf, P., Vissers, M. R., and Wang, Y., "MKID detector development for large scale far-infrared arrays," in [Millimeter, Submillimeter, and Far-Infrared Detectors and Instrumentation for Astronomy VIII], Presented at the Society of Photo-Optical Instrumentation Engineers (SPIE) Conference 9914 (June 2016).

[39] Werthimer, D., "The casper collaboration for high-performance open source digital radio astronomy instrumentation," in [General Assembly and Scientific Symposium, 2011 XXXth URSI], 1-4 (Aug 2011).

[40] McHugh, S., Mazin, B. A., Serfass, B., Meeker, S., O’Brien, K., Duan, R., Raffanti, R., and Werthimer, D., "A readout for large arrays of microwave kinetic inductance detectors," Review of Scientific Instruments 83, 044702 (Apr. 2012).

[41] Moncelsi, L., Ade, P. A. R., Angilè, F. E., Benton, S. J., Devlin, M. J., Fissel, L. M., Gandilo, N. N., Gundersen, J. O., Matthews, T. G., Netterfield, C. B., Novak, G., Nutter, D., Pascale, E., Poidevin, F., Savini, G., Scott, D., Soler, J. D., Spencer, L. D., Truch, M. D. P., Tucker, G. S., and Zhang, J., "Empirical modelling of the BLASTPol achromatic half-wave plate for precision submillimetre polarimetry," MNRAS 437, 2772-2789 (Jan. 2014).

[42] Pisano, G., Ng, M. W., Haynes, V., and Maffei, B., "A Broadband Metal-Mesh Half-Wave Plate for Millimetre Wave Linear Polarisation Rotation," Progress In Electromagnetics Research M 25, 101-114 (Aug. 2012).

[43] Pisano, G., Maffei, B., Ng, M. W., Haynes, V., Brown, M., Noviello, F., de Bernardis, P., Masi, S., Piacentini, F., Pagano, L., Salatino, M., Ellison, B., Henry, M., de Maagt, P., and Shortt, B., "Development of large radii half-wave plates for CMB satellite missions," in [Millimeter, Submillimeter, and Far-Infrared Detectors and Instrumentation for Astronomy VII], Proc. SPIE 9153, 915317 (July 2014). 